\title{
Artificial Intelligence - Now and the Future
}

\author{
Nwulu Evarista \\ Department of Computer Science, University of Jos \\ Jos, Nigeria
}

\begin{abstract}
The field of computer science has revolutionized every field of human endeavor. With the use of cleverly designed programs, computers have improved the quality and speed of productivity. Computers have become very useful machines, executing smart ideas rapidly. Artificial Intelligence seeks to take computing to further heights, building machines which do not just execute smart ideas, but think up smart ideas. This paper considers two recent achievements in the field of Artificial Intelligence, by rating their performance, in line with the goal of artificial intelligence.
\end{abstract}

\section{INTRODUCTION}

The first use of the phrase "Artificial Intelligence" in 1956 is attributed to John McCarthy of the University of Massachusetts [1]. It is a fairly new field which involves programming computers to play games, comprehend and respond to natural language, reproduce neural networks like those of humans and exhibit sensitivity (hear, see, move, react to the environment) like humans (this branch is termed robotics). First we must define Artificial Intelligence. A number of proposed definitions would be considered here.

"It is the science and engineering of making intelligent machines, especially intelligent computer programs."'[2]

"The ability of a computer or other machine to perform actions thought to require intelligence... An intelligent machine would be more flexible than a computer and would engage in the kind of "thinking" that people actually do". [3]

"A branch of computer science dealing with the simulation of intelligent behavior in computers."'[4]

"The capacity of a computer to perform operations analogous to learning and decision making in humans"[5]

It is also important to define intelligence in general:

“ The ability to learn facts and skills and apply them".[6]

"The ability to learn or understand things to deal with new or difficult situations"[7]

These generalized definitions give an idea, of what this field is concerned with. However an in-depth consideration of the definition of this field from the perspective of professionals in the field of computing and AI would be important. The last section of this paper considers the work of a foremost contributor to this novel field, Alan Turing.

In the last 15 years, researchers in the field of computer science have built machines, which have beat humans to competitions which are said to involve the use of intelligence. The two prominent machines in this category are IBM's Deep Blue and Watson. The next two sections would consider each of them respectively.

\section{THE RECORD BREAKING GAME BETWEEN DEEP BLUE AND KASPAROV}

\author{
Nahum Homti \\ Department of Computer Science, University of Jos \\ Jos, Nigeria
}

In 1997, Deep Blue computer designed by IBM defeated the 12 time chess world champion Garry Kasparov to a 6 round game of chess [8]. What was behind Deep Blue's victory? In an interview of IBM's Research Scientist Hsu by Ubiquity in 2005, Hsu admits to the use of "brute force" and pushing computational speed, as Deep Blue's edge over Kasparov. What does brute force involve? Claude Shannon researched on total possible moves for a typical game of chess [9]. He came up with a value of approximately 10120 moves. This leads to the idea of the game tree complexity. Where for every move an opponent makes(1), a computation is made of all possible moves which could be made in response(2), and the response as well of all the opponents possible responses to each of the computers response(3) moves as shown in the mini game tree below.

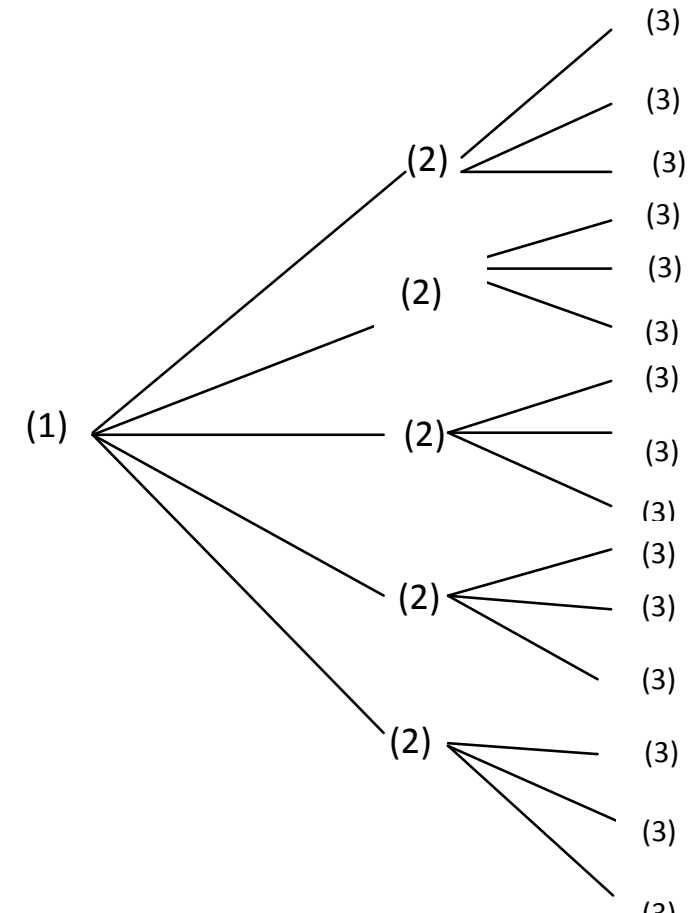

\section{FIG 1.0 A simple game tree}

For this simplified game tree in fig 1.0 after the first move (attacking) the response (defensive) move had 5 possibilities and in turn the response from the attacker had 3 possible moves for each of the 5 defense moves, making a total of 15 possible moves after the 3rd play. So for a typical game of chess of about 40 moves, using Shannon's approximation, at the end of the chess game tree there are 10120 possible moves [9].

How far down the game tree could Deep Blue go? Hsu [10] mentions that Deep Blue had a makeup of about 512 specially designed processors or chess chips, which had a combined search speed of about $2.0 \times 10^{8}$ positions per second. As gathered from IBM's website [8] Deep blue's average thinking time per game was 3.5 to 4 hours. This implies that 
Deep blue's thinking time on the game was averagely 252 seconds per move, thus coming to about $5 \times 10^{10}$ search positions per move. Compared to Shannon's approximation, it can be said that Deep Blue did not go very deep down the game tree, but compared with its human opponent, Kasparov who according to IBM [8] could search 3 moves per second, Deep Blue did have impressive depth. However, Deep Blue's win was a narrow one, considering it lost the first game, won the return match, drew the next 3 games and won the final deciding match. Despite its deep search, advantage it took a struggle to win Kasparov. So, Deep Blue lacked qualities that Kasparov had which, where experience and intuition [11].

Was Deep Blue Intelligent? "Does Deep Blue use Artificial Intelligence?" IBM gives a clear answer on its official website for answering FAQs(Frequently asked questions): "The long answer is no... and the short answer is no"[8]. Quoting the very words of Hsu [10] "... last year, going into the match... I did not really think we could win last year. This time I believe we can win. And that's a good feeling to have." Hsu uses the term "we" so Deep Blue got no credit, thus Deep Blue simply performed the instructions of intelligent research scientists, although at a remarkable speed.

What about Watson computer, giving smart answers to tricky quiz questions, was Watson intelligent? The next section would give attention to Watson.

\section{WATSON IN JEOPARDY}

"Tickets aren't needed for this "event" a black hole's boundary from which matter can't escape." What response is appropriate to the quoted statement? This statement was extracted from the 2011 Jeopardy show in which Watson participated and came off winner [12]. Statements are made, and the contestant who hits the response button first, provides the appropriate "question" to the given statement. The statements are drawn from a wide range of fields like history, geography, politics, arts, entertainment, science, archaeology, virtually every area of human endeavor. Thus the correct question for the aforementioned statement is "What is the Event Horizon"? Statements are posed in natural language, and the response "questions" are also given in Natural language. It must be admitted that this reversed statementquestion quiz is not simple nor is it trivial. Watson received the questions electronically, as a text file and gave its response verbally. Watson faced two strong opponents Ken Jennings (74 time Jeopardy champion) and Brad Rutter the 2001 Tournament of Champions, the Million Dollar Masters Tournament, and the Ultimate Tournament of Champions.[13][14]. Obviously Watson's victory was no small thing. What was the secret behind Watson's success? Can it be attributed to intelligence?

First, we need to consider Watson's hardware structure. As mentioned by the host of the 2011 Jeopardy show, Watson has 2 main units, each consisting of 5 racks, each rack has 10 powered IBM 750 servers. The equivalent of 2800 high speed computers tied together in a super high speed network (it can be compared to having a combined computer network of a standard university). It required a large refrigerating unit to keep it cool throughout the game [12]. On the stage was an Avatar (a representative of Watson) while the huge machine was kept behind the stage. Considering its database, according to Dunning [15] "Watson is built with a knowledge database of 200 million pages of raw text." Watson, no doubt was endowed with a vast wealth of information, as well as enormous hardware and hyper-speed processing, features to ensure it was unbeatable. But most importantly, what was
Watson's success algorithm? What was the underlying structure of its program? How did it assimilate the "statements" to produce just the correct "question", in "natural language"?

In producing Watson, IBM embarked on what it called "The DeepQA" project [16]. In its statement IBM mentions that Watson "is an application of advanced Natural Language Processing, Information Retrieval, Knowledge Representation and Reasoning, and Machine Learning technologies to the field of open-domain question answering. At its core, Watson is built on IBM's DeepQA technology for hypothesis generation, massive evidence gathering, analysis, and scoring". So DeepQA goes many steps further when compared with algorithms used by search engines. Search Engines, simply query data containing the relevant key words and extract the raw phrases containing those keywords, but as for the DeepQA strategy, on a given question, it has to sift the key words, which will be used in the query to fetch all matching data, next the data must be sorted to ensure highest priority or rating is given to the most relevant data, as well as the degree of confidence or precision of its response which is given in Natural Language [17]. DeepQA is a combination of thousands of algorithms IBM 2011b). Watson is a huge leap in the field of computer Question Answering. Prior to now, parsing data to a computer required strict adherence to syntactic and semantic rules to ensure input data is understood by the computer before processing. With the coming of Natural Language Processing (NLP), humans can communicate with computers in humanlike terms. The DeepQA research promises to have great benefits in research areas such as "business intelligence, technical support and health care, education" [18].

After considering Watson's outstanding performance in the Jeopardy show, can it be concluded that Watson is intelligent? For three years IBM researchers worked on 5500 independent experiments, each spanning about 2,000 CPU hours, to ensure the thousands of algorithms embedded within the software running Watson produces "their" thought-out result [19]. Watson delivered these results perfectly, however it added no new functionality or methodology or approach outside what its designers have embedded within it.

At this point we have seen impressive advancements made in this field of Artificial Intelligence, however we have not clearly, provided a guide to evaluate or ascertain a truly artificially intelligent machine. The final section would attempt to answer the question: What determines true Artificial Intelligence?

\section{TURING'S CONTRIBUTION IN THE FIELD OF ARTIFICIAL INTELLIGENCE}

British Mathematician Alan Turing (1912 - 1954) in his paper "Computing Machinery and Intelligence" published in 1950 in the journal "Mind", tried to find a means to test if a machine can think. This work is now popularly known as the Turing Test. What does the word think mean? In philosophy, psychology and neuroscience there are diverse definitions to this word. So in his work Turing first suggests, that since the words "think" and "machines" do not have one generally, clear and satisfactory definition, the question should be rephrased as "Can computers do what we (as thinking entities) can do?" [20]

Turing's test involves three participants in isolated rooms: a computer (which is being tested), a human, and a (human) 
judge. The human judge can converse with both the human and the computer by typing into a terminal. Both the computer and human try to make the judge think that they are the human. If the judge cannot consistently tell which is which, then the computer wins the game.

This test is indeed fundamentally useful, as it has been able to provide a means to rate or compare the behavior of machines with that of humans. Whereas in general, there is no clear definition of the words "thinking" and "intelligence" which can be applied to machines.

This test, though has been shown to have some weaknesses. As stated in the Wikipedia site[ 21] "Every element of this assumption has been questioned: the reliability of the interrogator's judgment, the value of comparing only behavior and the value of comparing it to a human." So the Turing test is subjective, if a single machine is tested by different judges, different conclusions would be made. This is not a desirable feature.

"The Turing test requires that the machine be able to execute all human behaviors, regardless of whether they are intelligent. It even tests for behaviors that we may not consider intelligent at all, such as the susceptibility to insults, the temptation to "lie" or, simply, a high frequency of "typing" "mistakes". If a machine cannot imitate these unintelligent behaviors in detail it fails the test" [22]. These are qualities known as human weaknesses and for a computer to pass the Turing test it should be able to exhibit these behaviors, should this be called "Artificial Intelligence" or "Artificial Weakness"? If a computer passes the Turing test does it understand the conversation it has had? This brings to fore the Chinese room thought experiment by John Searle, published in "Behavioral and Brain Sciences" in 1980 his paper "Minds, Brains and Programs." [23]

John Searle uses his thought experiment "The Chinese room" to reinforce his argument that a program cannot give a computer a mind, an understanding or a consciousness no matter how intelligently it may make it behave. Briefly, the Chinese thought experiment involves giving a non-Chinese speaking human, in a room, instructions (in English) on how to output accurately, Chinese characters, when given Chinese characters as input. If the instructions are clear enough and he is able to converse ( in writing ) with a Chinese speaking person (in another room) and can convince the Chinese speaker, that he or she understands Chinese, can it be said that he understands Chinese? Searle concludes that any computer programmed similarly to converse in Chinese does not understand Chinese.

Most people would take sides with Searle's opinion and strong argument. However, the possibility of an intelligent computer should not be ruled out. As it would be good to remember, there was a time when people where of the opinion that man can "never" fly. Although man has no avian wings, so to speak but he can board or fly a mechanical bird. Even though a computer has no brain cells likened to those of humans, a possibility of a computer imitating to some extent, human intelligence should not be cast aside.

To make progress on this subject, these two questions must be answered: What is intelligence? How should it be tested? In trying to define the goal of Artificial Intelligence Minsky [24] wrote: "Why can't we build, once and for all, machines that grow and improve themselves by learning from experience? Why can't we simply explain what we want, and then let our machines do experiments or read some books or go to school, the sorts of things that people do." On the possibility of educating machines Turing [20] mentions the possibility of designing child machines, which would be blank at birth, and would be filled up by educating it or learning by other means. However, Turing mentions that "the only problem would be that of programming". So researchers in the field of AI have the challenge of designing a machine, endowed with functions at birth (at design), and improves with growth like a child, and learns externally (from its environment) without any further capabilities bestowed (internally) on it by its designers. An interesting statement is made by Singh and Gupta [25]: "A true AI program should be able to perceive the world around it, autonomously decide its actions and should be able to adapt itself to the changes." This reflects the requirements of a machine to pass Schweizer's [26] Truly Total Turing Test (TTTT). So every new functionality acquired by the "intelligent computer" would be self derived, self intended. Such machines should be able to learn new languages like children simply by association, the machines should impress their builders with its new self "derived" functionalities, like a child impresses his parents with the new things he learns. These machines must be able to adapt new capabilities without the intervention of their designers. In this paper we want to introduce a Truly Thorough Total Turing Test (TTTTT(T5)) which not only involves the birth of child machines which can self improve and are self intended but are conceptually creative, innovative, instructive and inventive.

\section{CONCLUSION}

After considering this enhanced and broad test of intelligence, neither Watson nor Deep Blue has attained it. Computers at present have not formulated new concepts nor provided new solutions, they are still working within the confines of solving problems which humans have found solutions to or have defined algorithms to obtain their solutions. Computers are simply working within the confines of what they are fed with. So researchers in AI still have the task to design computers which would no longer be spoon fed with programs, but they will learn to "cook" so to speak their own programs, after gaining the necessary experience can formulate "recipes" to solve problems, which man at present has no solution to (introduce new concepts), to the amazement of its designers. Until this is achieved, computers would still remain as Fogel [27] said "preprogrammed to do exactly what humans want them to do. They accept input and generate the correct output. They may do it at blazingly fast speeds, but their underlying mechanisms depend on humans having already worked out how to write the programs that control their behavior". So future Artificial Intelligence seeks to give birth to "genius" computers. Which can reformulate the laws of physics as did Einstein, "discover" and "methodize" classical mechanics as did Sir Isaac Newton. Computers which can come up with breakthrough discoveries. AI also has a dream of producing "inventive" computers which can rival human inventors like Thomas Edison (inventor of the electric bulb), The Wright brothers (inventors of the aircraft), Ferdinand Verbiest ( inventor of the automobile) [28]. These computers (Future Artificial Intelligent computers) should also rival their designers (Future Artificial Intelligent computer Inventors) by conceiving concepts such as "designing" as well, thinking entities like themselves (the intelligent computers). However if computers are made to have intentions, what about undesirable intentions? Computers becoming evil geniuses as portrayed in some science fiction movies [29]. Who would be held responsible for their actions? This is not a forecast, to a downside to artificial intelligence, however this, brings to fore 
the various ramifications of intentions. Thus if future computers are given credit for breakthroughs, then they also will be held accountable for "undesirable actions". When computers become self decisive, self intended, conceptually creative, innovative then they have become truly intelligent, thinking outside their programmed box.

\section{REFERENCES}

[1] Webopaedia

(2014)

http://www.webopedia.com/TERM/A/artificial_intellige nce.html [accessed 26 Jun 2014]

[2] McCarthy, $\mathrm{J}$. http://www.formal.stanford.edu/jmc/whatisai/node1.html [accessed 3 Apr 2014]

[3] The

Free

Dictionary

http://www.thefreedictionary.com/artificial+intelligence [accessed 26 Nov 2011]

[4] Merriam-Webster (2011) http://www.merriamwebster.com/dictionary/artificial\%20intelligence [accessed 26 Nov 2011]

[5] Dictionary.com http://dictionary.reference.com/browse/artificial\%20intel ligence?s=t Encarta dictionary (2009)

[6] Microsoft Corporation, 1993 -2008.

[7] Meriam- Webster (2014)

[8] http://www.merriam-webster.com/dictionary/intelligence

[9] IBM (2011a) http://www.research.ibm.com/deepblue/watch/html/c.sht $\mathrm{ml}$ [accessed 3 Dec 2011]

[10] Shannon, C.E. (1950) "Programming a Computer for Playing Chess". Philosophical Magazine. Vol. 41.56275.

[11] Feng-Hsiung Hsu (2002) Behind Deep Blue, Princeton university Press, 2002

[12] MIT (2009) "Introduction to AI Techniques, Game Search, Minimax, and Alpha Beta Pruning”, SP. 268, page 15 ,

[13] Youtube (2011)

[14] Jeopardy show, http://www.youtube.com/watch?v=seNkjYyG3gI\&featur $\mathrm{e}=$ related[accessed 26 Nov 2011]

[15] Stauffer, C. (2002) "Manheim Township. Man back in 'Jeopardy!' in Million Dollar Masters Tournament". Lancaster New
[16] Toland, B. (2005) "A: He beat the best. Q: Who is Brad Rutter?" Pittsburgh Post-Gazzette. A1

[17] Dunning, H. (2011) IBM reveals secret to Watson's success. Canadian University Press Newswire http://cupwire.ca/2011/03/31/ibm-reveals-secret-towatsons-success/ [accessed 28/08/2014]

[18] IBM $(2011 b)$

http://www.research.ibm.com/deepqa/faq.shtml\#18 [accessed 3 Dec 2011]

[19] The AI Magazine http://www.aaai.org/Magazine/Watson/watson.php [accessed 28 Nov 2011]

[20] Canadian University Press (2011) http://cupwire.ca/articles/45278 [accessed 31 Nov 2011]

[21] Ferrucci, D., Brown, E., Chu-Carroll, J. \& Fanet, J. (2010)

[22] Building Watson: An Overview of the DeepQA project, pp. 77

[23] Turing, A. (1950) "Computing Machinery and Intelligence", Mind LIX (236): pp 433-460

[24] Wikipedia (2011c)http://en.wikipedia.org/wiki/Turing_test [accessed 1 Dec 2011]

[25] Saygin A. P., Cicekli I. (2002) "Pragmatics in humancomputer conversation", Journal of Pragmatics $34 \mathrm{pp}$. $227-258$,

[26] Searle, J. (1980) "Minds, Brains and Programs", Behavioral and Brain Sciences 3 pp. 417-457

[27] Minsky, M. L. (1991) "Logical Versus Analogical or Symbolic versus Connectionist or Neat versus Scruffy," AI Magazine, Vol. 12:2, pp. 35-5 1.

[28] Singh, V.K. \& Gupta, A.K. (2009) "Applied Computational Intelligence and Informatics". SACI '09. 5th International Symposium pp. 545 - 550

[29] Schweizer, P. (1998) 'The Truly Total Turing Test', Minds and Machines 8, pp. 263-272

[30] Fogel, D. (2006) Evolutionary Computation:Toward a New Philosophy of Machine Intelligence , pp. 1 - 32

[31] Burness T. (2001)Ultimate auto album: an illustrated history of the automobile. Iola, WI, Krause Publications, $1.503 \mathrm{p}$.

[32] IMDb(2011)http://www.imdb.com/title/tt0343818/ [accessed 5 Dec 2011] 